with pSS, a typical B-cell-mediated autoimmune disease. The elevated expression of CXCR3 on CD27 CD38 ${ }^{\text {low }}$ CD2 $21^{- \text {llow }}$ B-cells in AS patients is suggestive for active involvement in the inflammatory response. These findings are indicative of B-cell involvement in the pathogenesis of AS, against current dogma. REFERENCES:

[1] Ranganathan V et al. Nat Rev Rheumatol. 2017;13(6):359-367.

[2] Thorarinsdottir K et al. Scand J Immunol. 2015;82(3):254-261.

Disclosure of Interests: Rick Wilbrink: None declared, Anneke Spoorenberg: None declared, Suzanne Arends: None declared, Frans G.M. Kroese Speakers bureau: BMS, Roche, Janssen-Cilag, Consultant of: BMS, Grant/research support from: BMS, Gwenny M. Verstappen: None declared DOI: 10.1136/annrheumdis-2021-eular.2957

\section{POS0413 1 COMPREHENSIVE IMMUNE PROFILING OF PERIPHERAL BLOOD IN PSORIATIC ARTHRITIS (PSA) PATIENTS: EXPANSION OF INTERMEDIATE MONOCYTES AND DECREASEDT REG AND CD8T CELLS}

A. Grivas ${ }^{1}$, M. Grigoriou ${ }^{1}$, P. Katsimpri' ${ }^{2}$, P. Verginis ${ }^{3,4}$, D. Boumpas ${ }^{1,2}$ ${ }^{1}$ Biomedical Research Foundation of the Academy of Athens (BRFAA), Laboratory of Immune Regulation and Tolerance Autoimmunity and Inflammation, Athens, Greece; ${ }^{2}$ National and Kapodistrian University of Athens, 4th Department of Internal Medicine, Athens, Greece; ${ }^{3}$ Institute of Molecular Biology and Biotechnology, Foundation for Research and Technology, Institute of Molecular Biology and Biotechnology, Foundation for Research and Technology, Heraklion, Greece; ${ }^{4}$ University of Crete Medical School, Laboratory of Immune Regulation and Tolerance, Division of Basic Sciences, Heraklion, Greece

Background: Psoriatic arthritis (PsA) is a heterogeneous inflammatory arthritis that develops in a subset of patients with psoriasis. According to the current paradigm, cells of the innate and adaptive immunity interact with resident tissue fibroblasts mounting an inflammatory response via complex cytokine networks in the skin and joints in which type 1 and type $17 \mathrm{~T}$ cells play a dominant role. The abundance and relative contribution of other peripheral blood immune cells to disease pathogenesis as well the molecular signature of peripheral blood mononuclear cells and tissue fibroblasts remain ill defined.

Objectives: To comprehensively characterize immune cell subsets driving inflammation in the peripheral blood of patients with active PsA and their impact on psoriatic skin fibroblasts.

Methods: Peripheral blood was collected from PsA patients $(n=31)$ and age-/ sex-matched healthy individuals $(H I)(n=9)$, after informed consent. Psoriatic skin biopsies were acquired from a subset of 5 patients and $3 \mathrm{HI}$. All patients fulfilled the CASPAR criteria for the diagnosis and displayed peripheral polyarthritis of moderate- to high-disease activity. Patients' demographic and clinical data were recorded at time of sampling. Disease activity was assessed using the Disease Activity Index for Psoriatic arthritis (DAPSA) score. Skin psoriasis activity indices, enthesitis and dactylitis were also recorded. Peripheral blood mononuclear cells (PBMCs) were isolated by ficoll density gradient centrifugation. Flow cytometry was performed using a BD FACS-Aria-III and analyzed using FlowJo software. The antibody staining panel utilized aimed at the identification of the following immune cell subsets: Monocyte subsets (HLA-DR+ CD14+/- CD16+/-), Plasmacytoid dendritic cells (HLA- DR+ CD123+), T helper (CD4+), cytotoxic T (CD8+), regulatory T (CD4+ CD25+ CD127-) and B cells (CD19+). Statistical analyses were performed using GraphPad Prism software. Differences between groups were compared using unpaired T test for parametric data; Mann-Whitney and Kruskal Wallis tests for non-parametric data. The level of significance was set at $P<0.05$.

Results: 9 males and 22 females PsA patients are included (mean age 50 years and the mean disease duration 19.2 years for skin disease and 5.9 years for arthritis). The mean DAPSA score was 43.4 , suggestive of high disease activity, while $8(26 \%)$ patients displayed clinical enthesitis at time of sampling Flow cytometry analysis revealed aberrancies in peripheral blood immune cell populations. More specifically, PsA patients displayed a significant increase in intermediate monocyte subset (HLA-DR+ CD14+ CD16+) compared to $\mathrm{HI}$ with patients with clinical enthesitis demonstrating a more exaggerated expansion of intermediate monocytes compared to patients without enthesitis. A trend towards increased patrolling monocytes (HLA-DR+ CD14- CD16+) was also noted although this did not reach statistical significance. In contrast, both regulatory $T$ cells and cytotoxic CD8+ T cells were significantly decreased probably due to their selective migration at the sites of inflammation. RNA-seq from whole blood and skin fibroblasts from affected skin are in progress.

Conclusion: These data demonstrate significant expansion of intermediate monocytes -more pronounced in the enthesitis affected individuals- and decrease in $\mathrm{T}$ regulatory cells and T cytotoxic cells in PsA peripheral blood. Increased antigen presentation and co-stimulation mediated via intermediate monocytes in combination with their proangiogenic properties may contribute to disease pathogenesis REFERENCES:

[1] Veale, D. J. \& Fearon, U. The pathogenesis of psoriatic arthritis. The Lancet (2018) doi:10.1016/S0140-6736(18)30830-4.
Disclosure of Interests: None declared

DOI: 10.1136/annrheumdis-2021-eular.3540

\section{\begin{tabular}{|l|l}
\hline POS0414 INCREASED BDNF LEVELS AS A PREDICTOR \\
\hline
\end{tabular} OF CENTRAL SENSITIZATION IN PATIENTS WITH ANKYLOSING SPONDYLITIS}

I. Shapoval ${ }^{1}$, K. Zaichko ${ }^{1}$, M. Stanislavchuk ${ }^{1} .{ }^{1}$ National Pirogov Memorial Medical University, Vinnytsya, Ukraine, Chair of Internal Medicine \#1, Vinnytsya, Ukraine

Background: Ankylosing spondylitis (AS) is a chronic rheumatic disease that manifests itself in a range of inflammatory changes, severe pain and rapid progression with the development of osteoproliferation and the formation of ankylosis. Prerequisites are created for the emergence of signs of central sensitization. Scientists are growing interested in the role of the phenomenon of central sensitization. Central sensitization is present in almost half of patients with chronic pain (45\% SpA, 41\% RA, $62 \%$ OA and $94 \%$ of FM patients) [1]. Brain neurotrophic factor (BDNF) is involved in pain regulation mechanisms and the occurrence of central sensitization, but its role in patients with AS is poorly understood

Objectives: Our study aimed to determine levels of plasma BDNF in patients with AS and evaluate their role as central sensitization predictors in patients with ankylosing spondylitis.

Methods: We examined 143 patients with AS according to modified New York criteria (26 women and 117 men) with mean age $42.1 \pm 11.3$ years $(M \pm \sigma)$ and 35 persons of the control group, representative by age, sex. The content of plasma BDNF was determined at $8: 00$ and $20: 00$ by ELISA and calculated the daily average and morning/evening ratio - 8:00/20:00 BDNF index. All patients completed self-administered questionnaire Fibromyalgia Rapid Screening Tool (FiRST) to detect FM. FM was defined by a score $=>5 / 6$ by the FiRST. The study was conducted in compliance with bioethical standards. All data were analyzed using IBM Statistics SPSS 22.

Results: Among 143 patients with AS, there were 51 persons with FiRST $\geq 5$, indicating central sensitization and probable FM. In the group with AS mean scores $(\mathrm{M} \pm \sigma)$ of plasma BDNF levels were $962.5 \pm 357.2 \mathrm{pg} / \mathrm{ml}$ at $8: 00$ and $834.7 \pm 510.0 \mathrm{pg} / \mathrm{ml}$ at $20: 00$ compared to control group $(785.2 \pm 109.7 \mathrm{pg} /$ $\mathrm{ml}$ and $450.6 \pm 358.9 \mathrm{pg} / \mathrm{ml} ; \mathrm{p}<0.001)$. $36 \%$ AS patients were with + FiRST and had higher daily average and evening BDNF levels and a decreased 8:00/20:00 BDNF index. According to 8:00/20:00 BDNF index, we divide AS patients into 4 groups: 1st quartile (Q1) included people with BDNF index $<0.83$; 2nd quartile (Q2) - 0.83 - 1.15; 3rd quartile (Q3) - 1.16 - 2.49; 4th quartile (Q4) - > 2.49. Table 1 shows detailed information about FM's quantitative characteristics in patients with AS $(n=143)$ depending on the 8:00/20:00 BDNF index.

In patients with AS and + FiRST was registered an inadequate decrease in plasma BDNF levels in the evening, as evidenced by a decrease in the 8:00/20:00 BDNF index, which was combined with increased disease activity and poorer of the functional status.

The ROC analysis results showed that the 8:00/20:00 BDNF index at the cut-off point of 0.95 confirms the presence of central sensitization in patients with AS with a sensitivity of $86.2 \%$ and a specificity of $79.6 \%$. The AUC is 0.878 , which indicates a good quality of the model. Patients with the 8:00/20:00 BDNF index $<0.95$ are many times more likely to detect features of central sensitization (OR $=20.9 ; 95 \% \mathrm{Cl}: 8.66-50.39, \mathrm{p}<0.001)$.

Conclusion: Our results showed high diagnostic value to determine plasma levels of BDNF and to calculate 8:00/20:00 BDNF index as central sensitization marker in patients with AS with a sensitivity of $86.2 \%$ and a specificity of $79.6 \%$.

Table 1. Quantitative characteristics of FM in patients with AS ( $n=143)$ depending on the 8:00/20:00 BDNF index.

\begin{tabular}{|c|c|c|c|c|}
\hline \multirow[t]{3}{*}{ Parameters } & \multicolumn{4}{|c|}{ BDNF «8:00 / 20:00» } \\
\hline & $<0.83$ & $0,83-1.15$ & $1,16-2.49$ & $>2.49$ \\
\hline & Q1 $(n=37)$ & Q2 (n=35) & Q3 $(n=35)$ & Q4 $(n=36)$ \\
\hline FiRST $\left(M_{ \pm \sigma}\right)$ & $4.49 \pm 1,46$ & $4.23 \pm 1.42$ & $\underset{\substack{3.03 \pm 1.44 \\
\star \star \star}}{ }$ & $\underset{* \star 2.81 \pm 1.04}{* \star \star \#}$ \\
\hline $\mathrm{FiRST} \geq 5, \mathrm{n}(\%)$ & $24(64,9 \%)$ & $17(48.6 \%)$ & $\underset{* \star * \star \#}{(25.7 \%)}$ & $\underset{* \star \star \star \#}{(2.78 \%)}$ \\
\hline
\end{tabular}

Notes: 1. ${ }^{*}$ - statistically significant differences relative to $Q 1\left(^{*}-p<0.05 ;^{* *}-p<0.01 ;{ }^{* * *}-p\right.$ $<0.001) ; 2$. \# - statistically significant differences relative to $Q 2(\#-p<0.05)$.

\section{REFERENCES:}

[1] Guler, M. A., Celik, O. F., \& Ayhan, F. F. (2020). The important role of central sensitization in chronic musculoskeletal pain seen in different rheumatic diseases. Clinical rheumatology, 39(1), 269-274.

Disclosure of Interests: None declared

DOI: 10.1136/annrheumdis-2021-eular.3908 